\title{
On the Near-Wake of a Formula One Front Wheel
}

\author{
R. D. Knowles \\ A. J. Saddington \\ K. Knowles \\ Aeromechanical Systems Group \\ Cranfield University, Defence Academy of the United Kingdom \\ Shrivenham, Swindon, SN6 8LA
}

\begin{abstract}
Velocity measurements were made in the near-wake of the front wheel of a Formula One car using a laser Doppler anemometer. Tests were carried out at 50 percent-scale in a closedreturn, three-quarter open-jet wind tunnel. Three configurations were investigated to determine the effect of both the car, and an external wheel support strut, on the wheel wake. In each of the configurations the test Reynolds number, based on wheel diameter, was $6.8 \times 10^{5}$. Three components of velocity were measured at two planes less than one wheel diameter downstream of the wheel axis. These data were used to visualise the extent of the wake, the flow structures present and also the streamwise turbulence intensity. The presence of the car was found to alter significantly the structure of the wheel wake when compared to an isolated wheel. In addition, the external support strut was found to have less impact in the presence of the car than previous isolated wheel measurements had suggested.
\end{abstract}


Keywords: LDA, Formula One, Wheel Wake

\section{SYMBOLS}

$D$ wheel diameter

$f \quad$ focal length (of laser Doppler anemometer probe)

$t$ transit time (of seeding particle)

$u \quad$ velocity component in the $x$-direction

$u_{\infty}$ freestream velocity

$v \quad$ velocity component in the $y$-direction

$w \quad$ velocity component in the $z$-direction

$x \quad$ streamwise Cartesian coordinate (see Figure 1)

y crosswise Cartesian coordinate (see Figure 1)

$z \quad$ vertical Cartesian coordinate (see Figure 1)

$\tau_{i} \quad$ integral timescale

\section{INTRODUCTION}

There is an increasing amount of literature detailing experimental measurement of the wake of isolated wheels, particularly with application to single-seat race car aerodynamics. This has been augmented recently by several computational studies that have exploited advances in computational methods and the general reduction in the cost of computing to verify high-fidelity CFD models against this difficult, multi-faceted flow problem. 
Early experimental work suffered from a lack of availability of rolling roads and consequently developed novel solutions to allow rotating wheels with static ground planes. Morelli [1] overcame some of the problems by allowing the rotating wheel to protrude through the ground plane, thereby ensuring that no physical contact was made. Stapleford and Carr [2] and Cogotti [3] positioned a rotating wheel a small distance from the ground and then sealed the resultant gap with flexible / low-friction materials. Comparison of the results of these two methods confirmed that any small gap between the wheel and ground is sufficient to reverse the, now accepted, positive lift vector acting on the wheel. The lack of moving-ground wind tunnels at that time drove work to establish whether, for road-vehicle aerodynamic development, a stationary wheel could provide a sufficient proxy for the real-life rotating alternative.

Probably the most comprehensive early experimental work on isolated wheel wakes was that of Fackrell [4] and Fackrell and Harvey $[5,6]$ which gathered surface pressure and wake pressure data for both a stationary wheel and a rotating wheel (with moving ground). In addition, the work also demonstrated the sensitivity of wheel flow fields to tyre shoulder geometry, albeit performed on a non-deformable tyre. Of particular significance was the theoretical proposition that the convergence of moving surfaces (rotating wheel and moving ground) upstream of the tyre contact patch would generate significant viscous action and eject a jet from this region. The proposition was made to explain a significant pressure peak that was observed in front of the contact patch, which was entirely absent from the stationary case. With these measurements Fackrell [4] was the first to demonstrate that both ground motion and wheel rotation were essential for the investigation of isolated wheel flows. Several studies subsequently confirmed that the same conditions 
were also essential for correct full-vehicle simulation. These studies included research at Ford by Hackett et al. [7], Imperial College, London by Bearman et al. [8] and General Motors by Mercker and Berneburg [9], Mercker et al. [10], all on road car geometries. Later work by Wildi [11] and Mueller et al. [12] at Porsche confirmed the significance of ground motion and wheel rotation on race car aerodynamics.

One area of experimental work looked to address the problem of wheel lift measurement, which is complicated by mechanical ground contact. Stapleford and Carr [2], Fackrell [4] and Cogotti [3] calculated wheel force by measuring the surface pressure acting on the rotating surface of the wheel and integrating it. Latterly Hinson [13], Skea et al. [14] and Mears [15-18] all made measurements using the pressure integration method with higher-fidelity pressure measurement systems. Each investigator used a different wheel geometry, therefore quantitative comparison is impossible, but their results do agree qualitatively. In particular, Hinson and Mears both identified a significant negative pressure peak downstream of the contact patch. This was proposed to be generated by the rapid divergence of the wheel and road surfaces, the inverse of the action on the upstream side of the contact patch proposed by Fackrell [4].

In recent years, the advent of steel-belt rolling road systems has allowed direct measurement of wheel forces by load cells positioned beneath the belt, directly below the tyre contact patch, as used by Wäschle et al. [19]. Whilst this is becoming increasingly common in automotive development, the authors are unaware of further published literature on this topic. The remaining experimental work has focussed on providing measurements of wheel wakes to determine the structure and interactions at work. 
An in-depth wake survey was conducted by Nigbur [20], who measured three-components of velocity in the wake of a 50 percent-scale Formula One wheel using a hot-wire anemometer. Ten streamwise planes were measured, with the data presented as time-averaged contours of each component, plus contours of the associated root-mean-square (RMS) fluctuations. Analysis of the vortical structures in the wake was difficult as the spanwise and vertical velocity components were presented individually, rather than as in-plane vectors. The streamwise data were somewhat more informative, confirming the distinctive wake profile identified by Fackrell [4] (inverted-T or $\perp$ in the vertical plane perpendicular to the stream wise direction). The data also exhibited regions with high RMS values, particularly downstream of the support strut. No regions of reversed flow could be identified in the wake as the anemometer was insensitive to the direction of the streamwise velocity component. The wake was notably asymmetric which was attributed to ineffective boundary layer suppression by a small moving ground that was approximately the same size as the wheel.

Further studies of the wake of isolated race car wheels were reported by Knowles [21-24], Mears [15-18] and Wäschle et al. [19]. Knowles and Wäschle et al. used laser Doppler anemometry (LDA), whilst Mears et al. used particle image velocimetry (PIV), both sensitive to flow direction. All authors measured asymmetric wake structures, particularly in the vertical plane perpendicular to the streamwise direction, which featured combinations of trailing vortices, dependent on test geometry and measurement location. Recently Issakhanian et al. [25] produced PIV measurements in the wake of a similar isolated race car wheel which confirmed the observations of the previous authors whilst also providing valuable validation data for their subsequent computational research. 
Early computational fluid dynamic wheel simulations, such as those performed by Skea et al. [26], Axon [27], Basara et al. [28], Knowles [23], Mears et al. [17] were predominantly steadystate, finite-volume simulations using various Reynolds-Averaged Navier-Stokes (RANS) codes and turbulence models. The various turbulence models and numerical schemes all failed to accurately predict the main forces on the wheel. In the majority of cases this was due to incorrect prediction of flow separation from the tyre. In general these investigations did not greatly add to the information provided by the experimental literature. The Lattice-Boltzman simulation carried out by Wäschle et al. [19] improved lift prediction but drag forces were still in error, suggesting that the flow field remained incorrect. More recently two authors have published the results of significantly higher-fidelity computational models than those previously mentioned. McManus and Zhang [29] extended previous RANS work presenting results from Unsteady RANS (URANS) simulations of the experiments of Fackrell [4]. The work clearly visualised the flow features postulated by Fackrell from his experimental data and also extended the wake schematic previously proposed by Knowles [23]. As with the previous RANS-based computational studies, the simulations struggled to predict the lift and drag forces acting on the wheel.

Axerio et al. (2009 and 2012) reported simulations of the experimental work of [25] comparing several turbulence modelling approaches and found significantly improved prediction of both the flow field and vortex dynamics using URANS (k- $\omega$ Shear Stress Transport) and Large Eddy Simulation. This work was, however, performed on a stationary wheel, which is perhaps less computationally challenging, due to the different predominant flow features. Force predictions were not compared to experimental values. 


\section{AIMS AND OBJECTIVES}

The aim of this study was to outline the wake of a Formula One wheel in the presence of a Formula One car and identify the main areas of difference between that and an isolated wheel. In addition the study aimed to illustrate the effect of an external support strut in the presence of the car. The objective of the study was to generate three-dimensional mean velocity data in a region less than one wheel diameter downstream of the wheel axis, termed the near-wake.

\section{EXPERIMENTAL SET-UP}

The following description of the experimental set-up is broken down into three main sections: the wind tunnel; the test components; and the anemometer.

\subsection{Wind Tunnel}

Cranfield University's D.S. Houghton wind-tunnel at the Defence Academy of the United Kingdom in Shrivenham was used for all testing. It is a $2.74 \mathrm{~m}$ by $1.66 \mathrm{~m}$ closed-return, three-quarter open-jet wind tunnel (Figure 1) equipped with a continuous-belt rolling road system. The movingground, synchronised with the tunnel freestream velocity, provided both boundary layer suppression and wheel rotation. The wind-tunnel ground boundary layer was removed by the application of suction immediately upstream of the rolling-road. The level and distribution of the suction were optimised during wind tunnel commissioning, and coupled with a knife-edge transition to the belt, ensured minimal belt boundary layer. A vertical profile of total pressure (normalized to the tunnel 


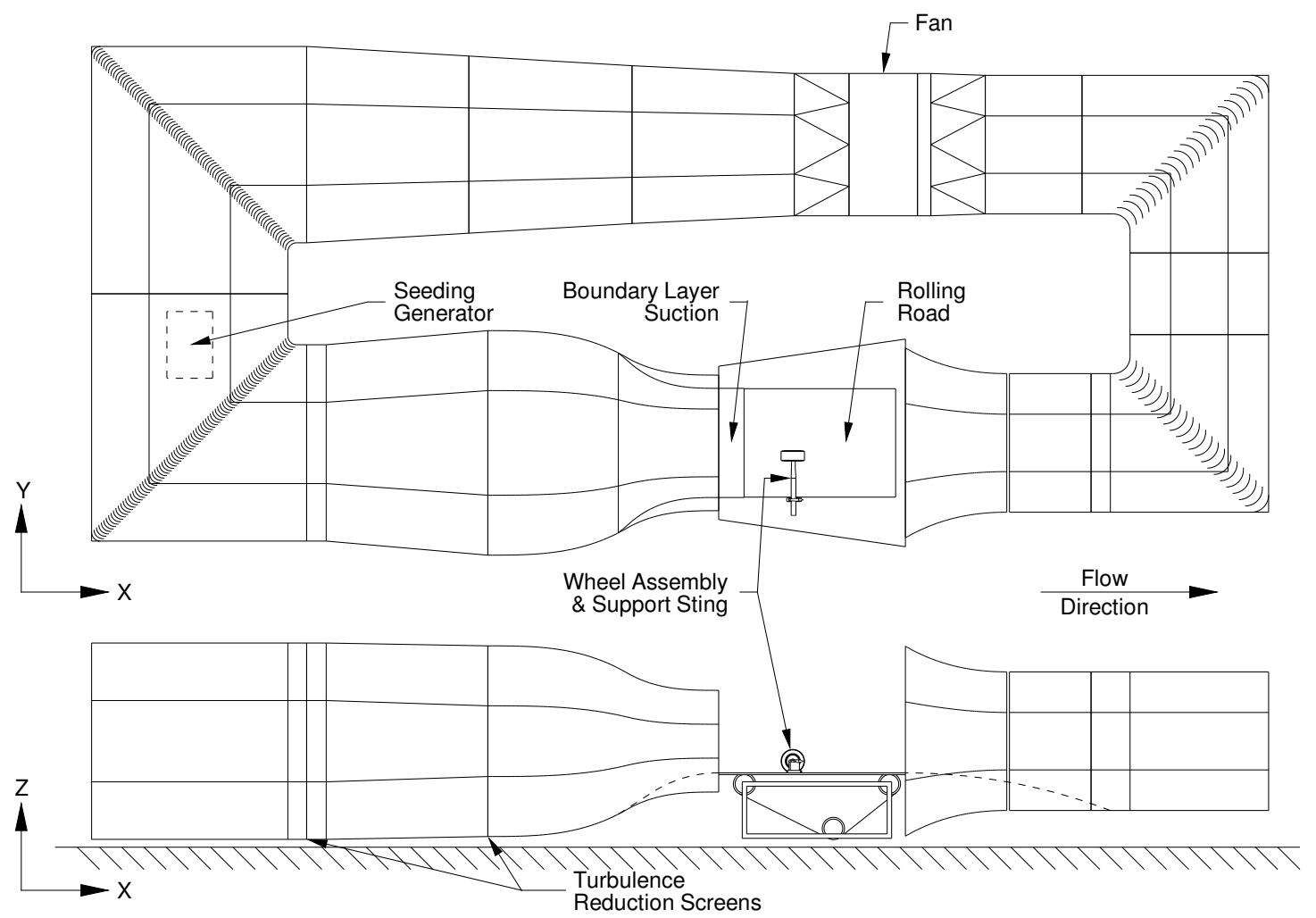

Fig. 1: Schematic representation of the test configuration.

centreline value) is shown in Figure 2 and reveals a small total pressure gradient, which rose by approximately $0.7 \%$ as the belt was approached. The minimum recorded total pressure was $99.76 \%$ of the freestream value, occurring $0.754 \mathrm{~mm}$ above the surface of the belt. The action of the aerodynamic loads from both the wheel, and more significantly, the model car were counteracted by the application of distributed suction to the underside of the rolling-road belt. The temperature of the air and rolling-road surface were held constant $\left(25^{\circ} \mathrm{C} \pm 0.5^{\circ} \mathrm{C}\right)$ throughout testing by active temperature control. Further details of the wind tunnel, rolling road and calibration can be found in Finnis et al. [32]. 


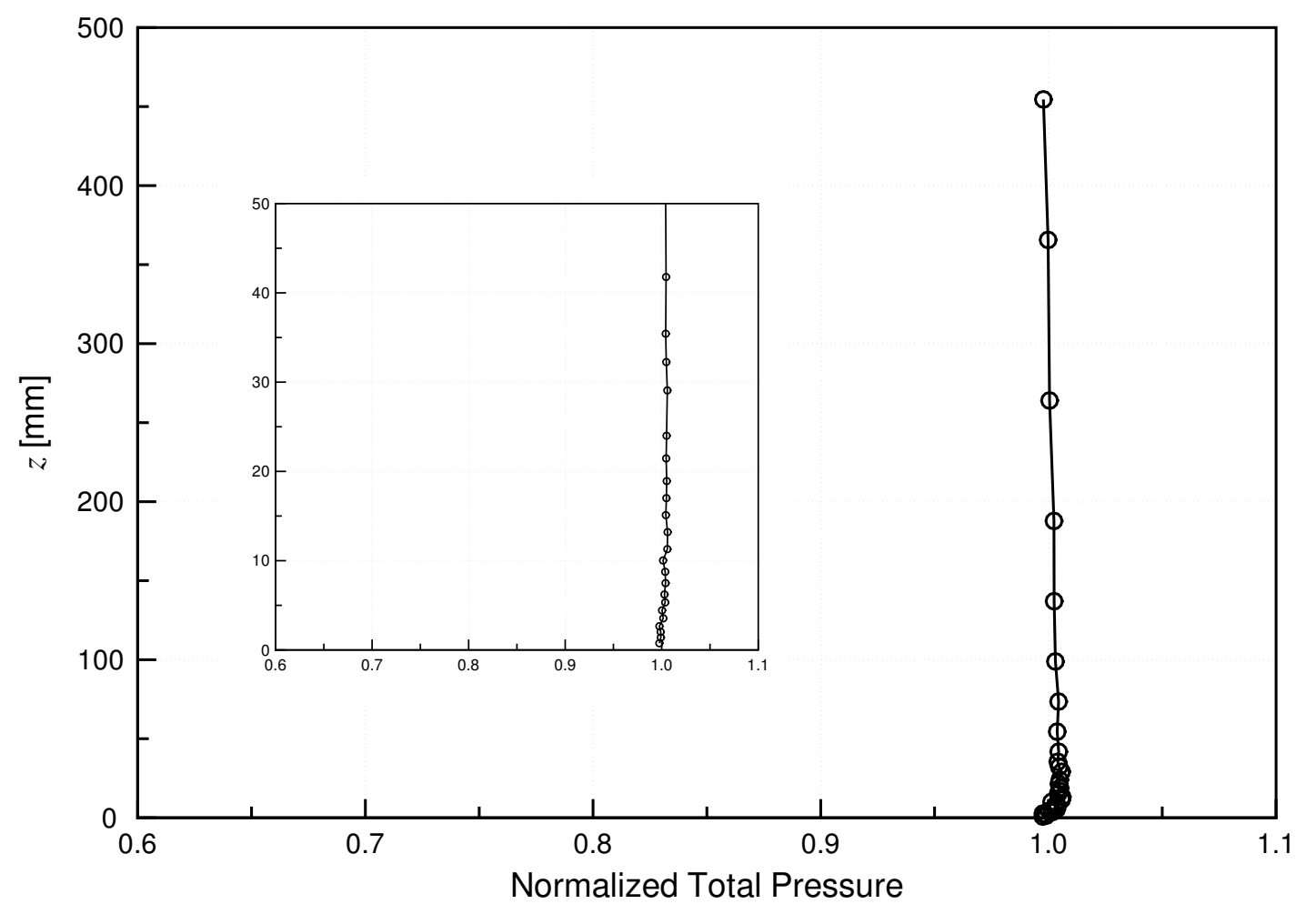

Fig. 2: Boundary layer profile at the wheel test location. 


\subsection{Test components}

All of the components represented the state-of-the-art when tested having been employed in the development of Formula One cars from 2000 onwards. The following sections describe the wheel, external support sting and car model. Three combinations of these components were tested: wheel and sting; wheel, sting and car and wheel and car. Comparison of these three test configurations allowed the effect of the car and the effect of the external support sting to be separately evaluated.

\subsubsection{Wheel and sting assembly}

The 50 percent-scale Formula One front wheel assembly is shown in exploded detail in Figure 3. The figure illustrates the hub spoke pattern and the shape of the non-deformable, carbon-fibre tyre, including the grooves on its surface. Flow through the hub is restricted by the presence of the brake rotor and suspension upright. The brake rotor was mounted to the hub and rotated with it, the radial channels in the disc acting as a simple centrifugal pump. The suspension upright was mounted directly to either the wheel support strut or car suspension (depending on test configuration) and therefore remained stationary (non-rotating). The upright was produced using stereolithographic sintering and featured an intricate spoke pattern which allowed limited air passage.

The support sting featured a symmetrical aerofoil cross-section (thickness-to-chord ratio of 0.39) which (see Figure 1) was mounted beside the rolling road such that the wheel position remained constant throughout the test configurations. No additional vertical force was applied to the wheel. Regular checks were performed with a stroboscope and optical tachometer to confirm that wheel vibration was minimal and that the rotational speed of the wheel matched the belt/wind 


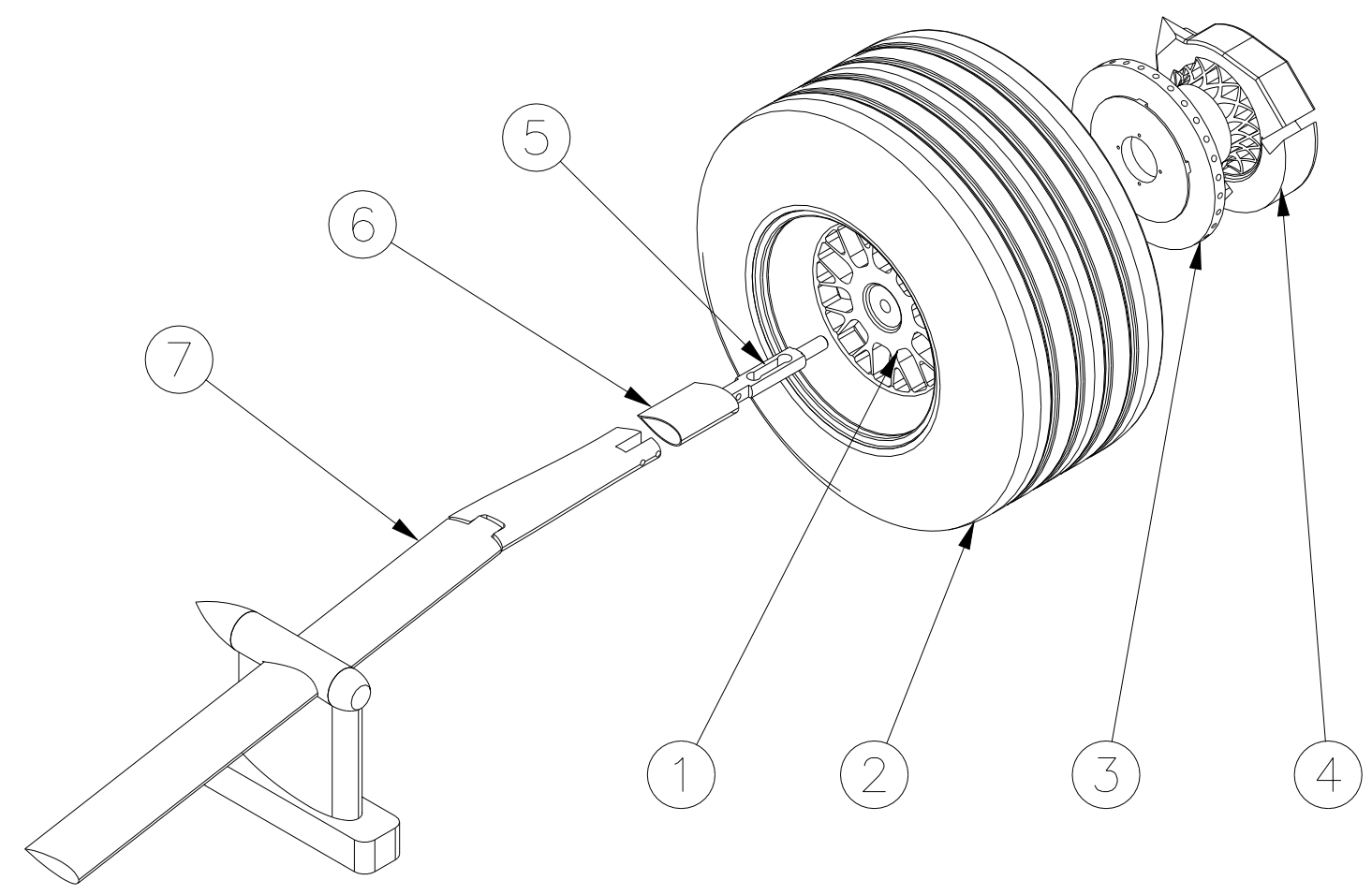

Fig. 3: Formula One wheel assembly and support sting.

Key:

4 = Suspension Upright

$1=$ Wheel Hub

$5=$ Wheel Drag Loadcell

$2=$ Non-Deformable Tyre

$6=$ Loadcell Shroud

$3=$ Brake Rotor

$7=$ Support Sting 


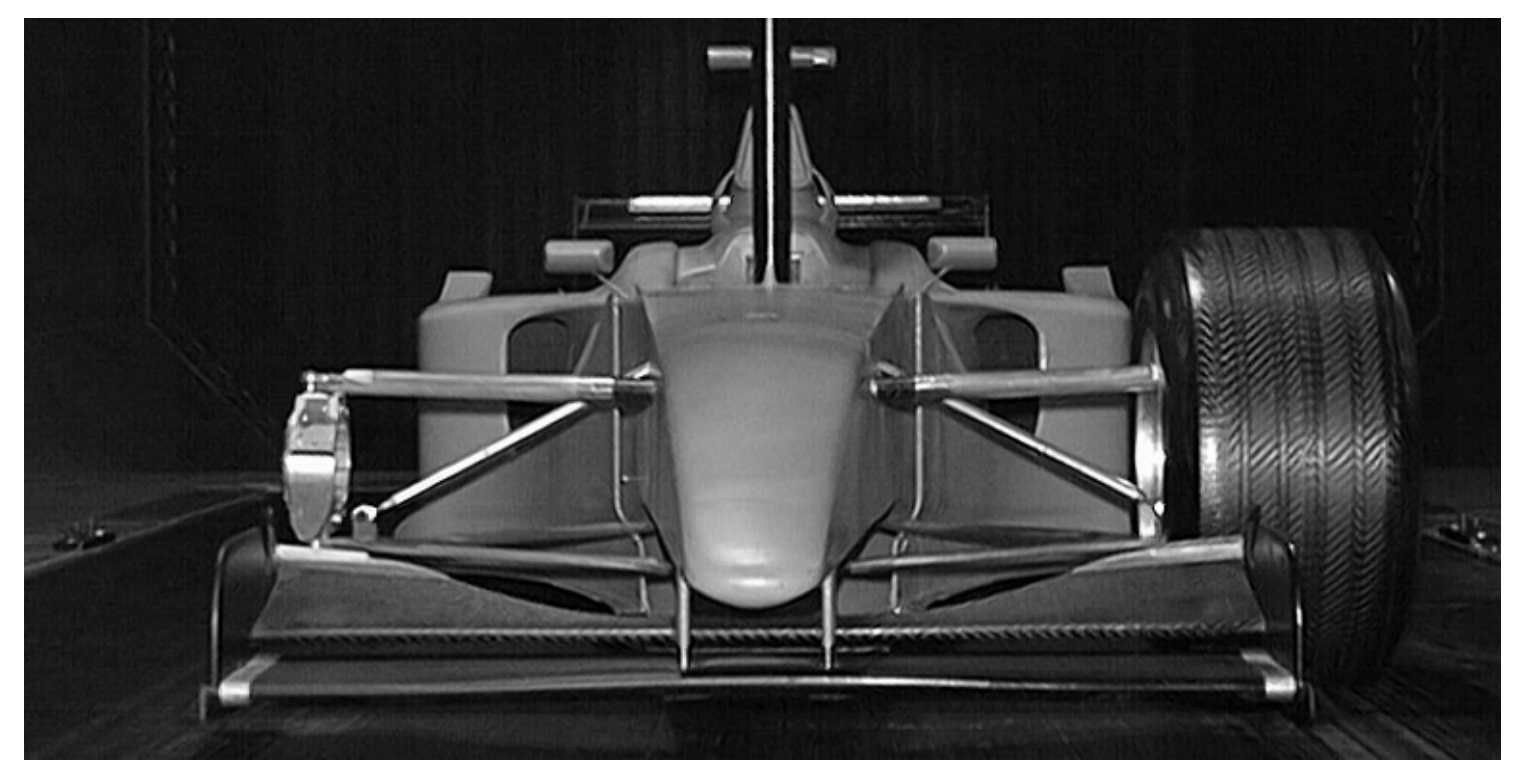

Fig. 4: Installed Formula One car model.

speed.

\subsubsection{Formula One Car}

Two of the three test configurations featured a fully-detailed, 50 percent-scale model Formula One car. The car was suspended from the tunnel overhead support strut in close proximity to the road. The car parameters (ride-height, pitch angle, front wing configuration etc) were fixed for all of the tests. Only one wheel assembly was used, supported either by the sting or the car suspension depending on the test configuration. The lack of the other three wheels was assumed to have negligible effect in this configuration, although this assumption was not experimentally verified. A photograph of the test configuration is shown in Figure 4. 


\subsubsection{Laser Doppler Anemometer}

The laser Doppler anemometer used in this study was a Dantec FibreFlow system operating in back-scatter mode. The system included a frequency shift on one of each of the beam pairs (acheived by a Bragg cell) to allow determination of flow direction as well as magnitude. Ordinarily, this system would be operated in coincidence mode, collecting three-component velocity data. In this case, the tunnel layout required a probe focal length greater than $2 \mathrm{~m}$, and only one such lens was available. The single, $f=2.5 \mathrm{~m}$ probe could collect two velocity components simultaneously, however the mean data were improved by collecting a single velocity component per test and repeating each test three times to gather $u, v$ and $w$ data. A shorter focal-length probe would have enabled simultaneous measurement of the three velocity components but placed the probe body in the wind-tunnel jet and effectively negated the non-intrusive benefit of the LDA technique.

The signal-to-noise ratio of the probes operating in back-scatter mode was found to be adversely affected by the reflection of laser light from the test components. In particular, the reflections from the light-coloured barge-board of the car affected approximately one quarter of each measurement plane. It was not possible to remove the reflections completely, but the application of matt, fluorescent-magenta tape to the car doubled the data rate and increased the signal-to-noise ratio. Fluorescent magenta was found to be more effective than conventional matt black or red as it actively absorbed some laser light during fluorescence reducing the amount of light reflected at the incident wavelength. 
Flow seeding was provided by a JEM Hot2000 fog generator positioned inside the wind tunnel duct (see Figure 1). The wake of the seeder was not detectable in the working section as it was dissipated following passage through a set of cascades, three turbulence-reduction screens and the boundary-layer suction. The seeder produced high-volume, ambient-temperature seeding which was continuously generated throughout all tests. An 85:15 water-ethylene-glycol mix was used to reduce the volatility of the seeding, which had a mean particle size of $1.3 \mu \mathrm{m}$.

\subsection{Test procedure}

All testing was carried out at a freestream velocity of $30 \mathrm{~ms}^{-1}$, which yielded a Reynolds number, based on wheel diameter, of $6.8 \times 10^{5}$. Reynolds number effects were not investigated in this study. The mean freestream turbulence intensity, without the test components, was $0.25 \%$.

Figure 5 illustrates one of the measurement planes in the wake of the wheel. Each plane was $0.34 \mathrm{~m} \times 0.34 \mathrm{~m}$ and comprised 613 data points, arranged in a $\mathrm{y}-\mathrm{z}$ planar grid, perpendicular to the freestream. The spatial resolution of the measurement plane $(20 \mathrm{~mm}$ and $10 \mathrm{~mm}$ in the spanwise and vertical directions, respectively) was chosen to ensure that the measurement volume at each point did not overlap that of other points. Two measurement planes were extracted for each test configuration, positioned 0.75 and 1 wheel diameter downstream of the wheel axis.

The stochastic nature of LDA sampling meant that, in order to limit the time required to complete a measurement plane, the maximum sampling time $(20 \mathrm{~s})$ and maximum number of samples (5000) was held constant for each data point. The data acquisition would move to the next point on breach of either condition. These conditions attempted to maximise the sample population whilst 


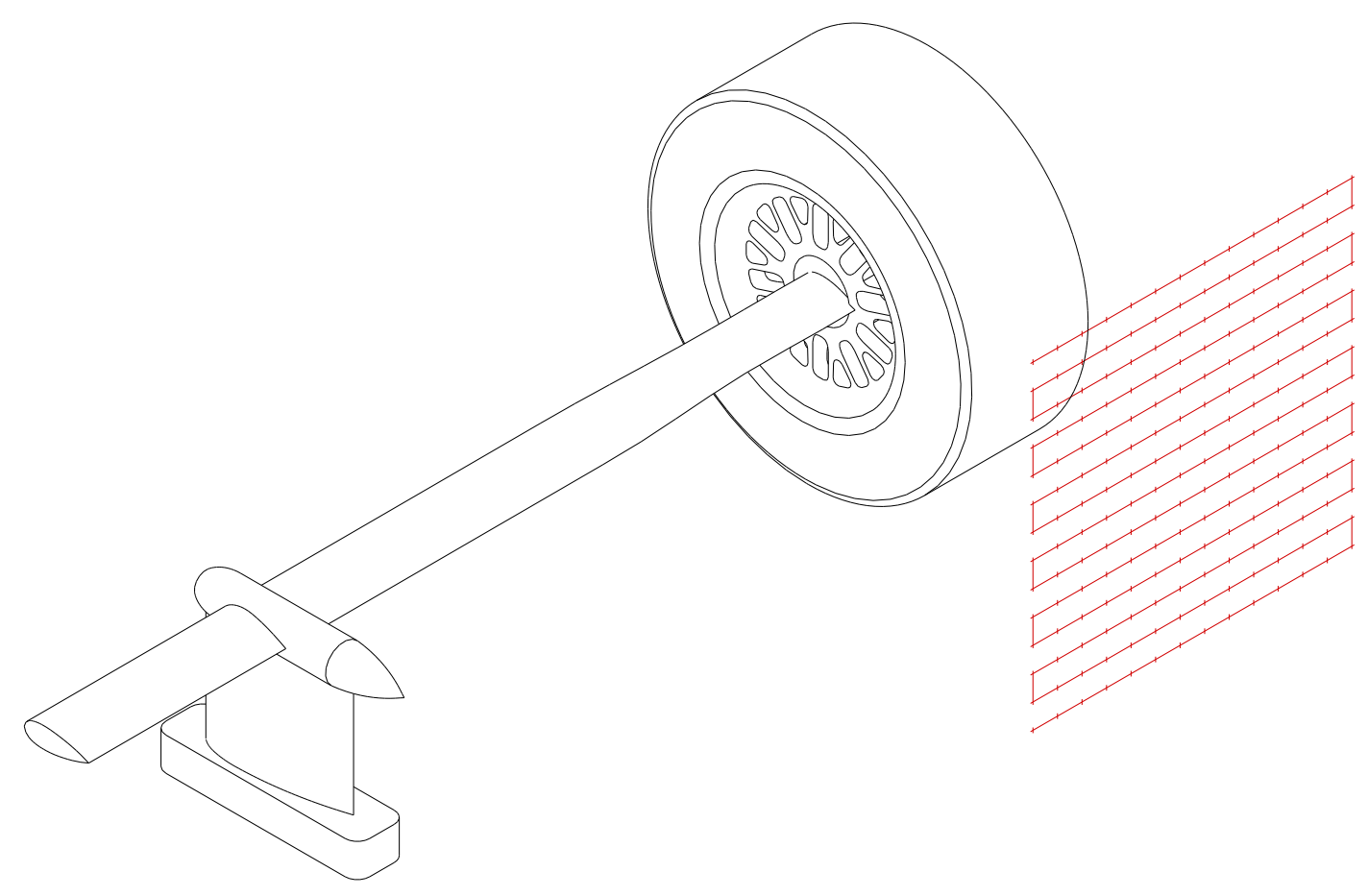

Fig. 5: Example measurement plane $(0.34 \mathrm{~m} \times 0.34 \mathrm{~m}$, comprising 613 data points $)$. 
avoiding potentially unlimited acquisition time. A five second pause was inserted after each probe movement to allow any probe vibration to decay.

\section{UNCERTAINTY ANALYSIS}

\subsection{Wind and Ground Speed}

Freestream air velocity was maintained to within $\pm 0.2 \%$ of the target wind speed by the wind tunnel control system, whilst the rolling-road belt speed was governed to within $\pm 0.07 \%$ of the same target.

\subsection{Positional Uncertainty}

The passage of a particle through any part of the LDA measurement volume would trigger a velocity measurement and as such the exact location of any measurement is unknown. The physical extents of the measurement volume was known and therefore the maximum positional error was one half of the volume's dimensions, $\pm 4.1 \mathrm{~mm}$ streamwise, $\pm 5.5 \mathrm{~mm}$ spanwise and $\pm 1.5 \mathrm{~mm}$ vertically.

\subsection{Seeding response}

The response of a seeding particle to flow accelerations was analysed to provide an estimate of the ability of the particles to accurately track the flow. The analysis was based on the method of Dring [33], and involved calculation of the seeding Stokes number. Dring determined experimentally that for low Stokes numbers $(<0.01)$, the maximum velocity error is equal to the seeding Stokes 
number. The Stokes number, and therefore maximum velocity error in this case, was estimated as $6.4 \times 10^{-4} \mathrm{~ms}^{-1}$.

\subsection{Velocity bias}

Continuous sampling meant that individual LDA measurements were triggered by the transit of any particle through the measurement volume and introduced a velocity bias. It follows that a higher velocity would lead to more observations than a lower velocity, biasing the raw mean of a set of observations to be higher than the true mean velocity at that location. The transit time of each particle through the measurement volume was also recorded for each measurement and it's inverse provided a convenient weighting to produce an unbiased estimate of the mean at each location. This method of bias correction was originally proposed by Buchave et al. [34].

\subsection{Sampling error}

LDA measurement is a stochastic process and therefore the collection of statistically independent samples is difficult. As previously mentioned, the sampling rate is partly determined by the local flow conditions and thus varied with measurement location. It follows therefore, that the uncertainty also varied with location. The uncertainty was estimated using the method of Benedict and Gould [35] based on the integral timescale, $\tau_{i}$, which was in turn estimated using a method outlined by Nobach [36]. It was subsequently determined that, to a $95 \%$ confidence level, $30 \%$ of the measurement points had an error of less than $1 \%$ with the remainder having an error less than $10 \%$. 


\section{RESULTS AND DISCUSSION}

Data from two planes (0.75 D and 1.0 D downstream of the wheel axis) are presented for three configurations, labeled F1 Wheel - Aluminium Sting, F1 Wheel \& Car - Aluminium Sting and F1 Wheel \& Car - No Sting. comparison of the first two gives an indication of the effect of the car on the wheel wake whilst comparison of the latter two gives an indication of the effect of the support sting in the presence of the car. The effect of the car on the wheel wake will no doubt be dependent on the particular car configuration, front wing span, downforce level etc. and likewise the effect of the sting has been shown to be dependent on its particular geometry [23].

\subsection{Streamwise Velocity Contours}

The contours of streamwise velocity (Figure 6) effectively outline the shape and extent of the velocity deficit in the wake of the wheel. Comparison of the structures of these wakes clearly shows that the car has a marked impact on the wake structure. Firstly, the central portion of the wake, above wheel-axle height, has changed significantly in the presence of the car. Whilst this section of wake was contained within the projected profile of the tyre for the isolated wheel wake, the presence of the car has increased the overall height of the wake by approximately one-third and increased the width to approximately that of the tyre. The increase in height suggests that the point at which flow separates from the tyre surface has moved upstream in the presence of the car. The increased width suggests that the two upper vortex structures, which reduce the width of the wake by entraining freestream air and are prevalent in the literature for isolated wheels, may not 
be present in the wheel-and-car configurations.

Secondly, the distribution of the reversed flow regions (delineated by the dashed lines) are also significantly affected by the presence of the car. The higher central position of the main reversed flow region may suggest that the entrainment of flow into this region, usually provided by the upper vortices, has been replaced by a strong recirculation with an approximately vertical axis and almost entirely contained within the projected profile of the wheel. This containment within the profile may somewhat explain the lack of advection of the reversed flow regions in the presence of the car which are no longer present by one diameter downstream. Comparison of the two configurations that feature the car suggest that the external support strut has little effect in terms of the overall wake shape, except for a slight change in the magnitude and distribution of the reversed flow regions.

\subsection{In-Plane Velocity Data}

The in-plane velocity data are presented as both vectors to give comparison of magnitudes (Figure 7) and also using the line-integral convolution (LIC) technique which improves the visualisation of the wake structures (Figure 8). The output of the LIC technique is similar to an oilflow image, producing a monochrome, constant-length streamline plot of very high density. Streamlines are traced from all points in a velocity field and often reveal structure which are not immediately apparent in conventional velocity vector plots. The LIC plots are best interpreted in conjunction with velocity vector plots as the LIC plots contain no velocity magnitude or directional information. More information on the LIC implementation used for this work can be found in an earlier 

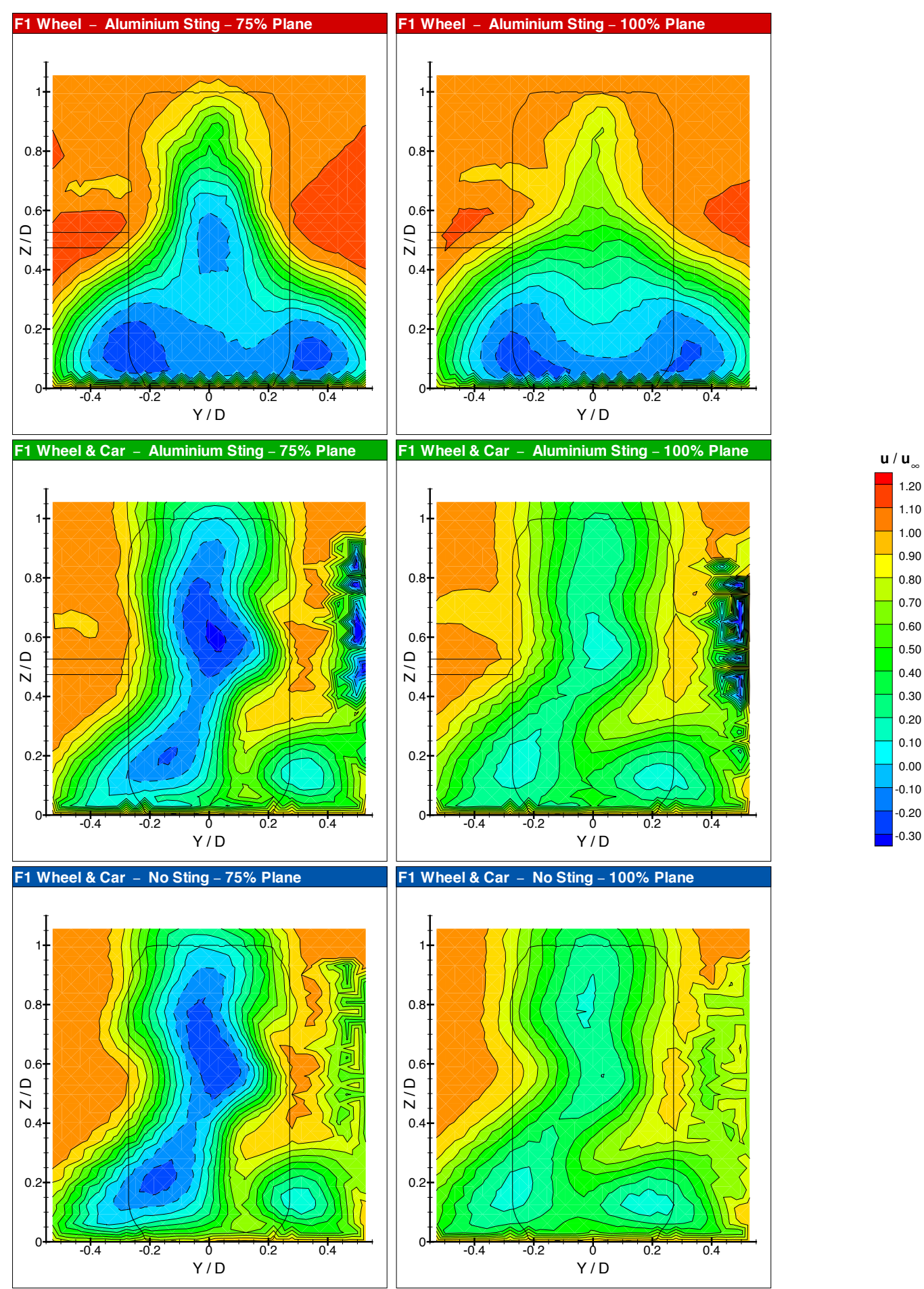

Fig. 6: Contours of mean $u$ velocity for $x / D=0.75$ and 1.0 at three test configurations (reversed 20

flow regions bounded by dashed lines). 
paper by the author [37].

The in-plane velocity data for the isolated wheel clearly show the two trailing vortices that have been observed in the wake of isolated wheels since the early work of Fackrell [4]. However, in the presence of the car there are very few coherent wake structures.

The strong central downwash region has been replaced by in-wash which, as revealed by the velocity contours, turns towards the wheel to generate the reversed flow rather than downwards in the isolated case. It was proposed earlier that this was due to the lack of vortices to entrain flow into this region. The velocity vectors do not feature the large trailing vortices associated with isolated wheels. There exists a single, relatively small trailing vortex on the inboard side of the wheel. Both Knowles [23] and more recently, Axerio-Cilies et al. [31] observed that the centre of the isolated-wheel vortices tended to remain within the projected profile of the wheel. Therefore as this vortex has its origin outside the wheel profile it may suggest that it is not generated by the wheel itself. The work of van den Berg [38] on wing-wheel interaction suggests that the structure has the correct sense and is on the likely track of the front-wing endplate vortex.

The lack of coherent structures in the wheel wake when the car is present make it difficult to assess the impact of the support strut. However, as with the previous velocity contours, the sting appears to have had little quantifiable effect in the presence of the car.

\subsection{Streamwise Turbulence Intensity}

Comparison of the contours of streamwise turbulence intensity (Figure 9) reveal similar differences to those outlined for the velocity contours. One item of note however, is the effect of the 

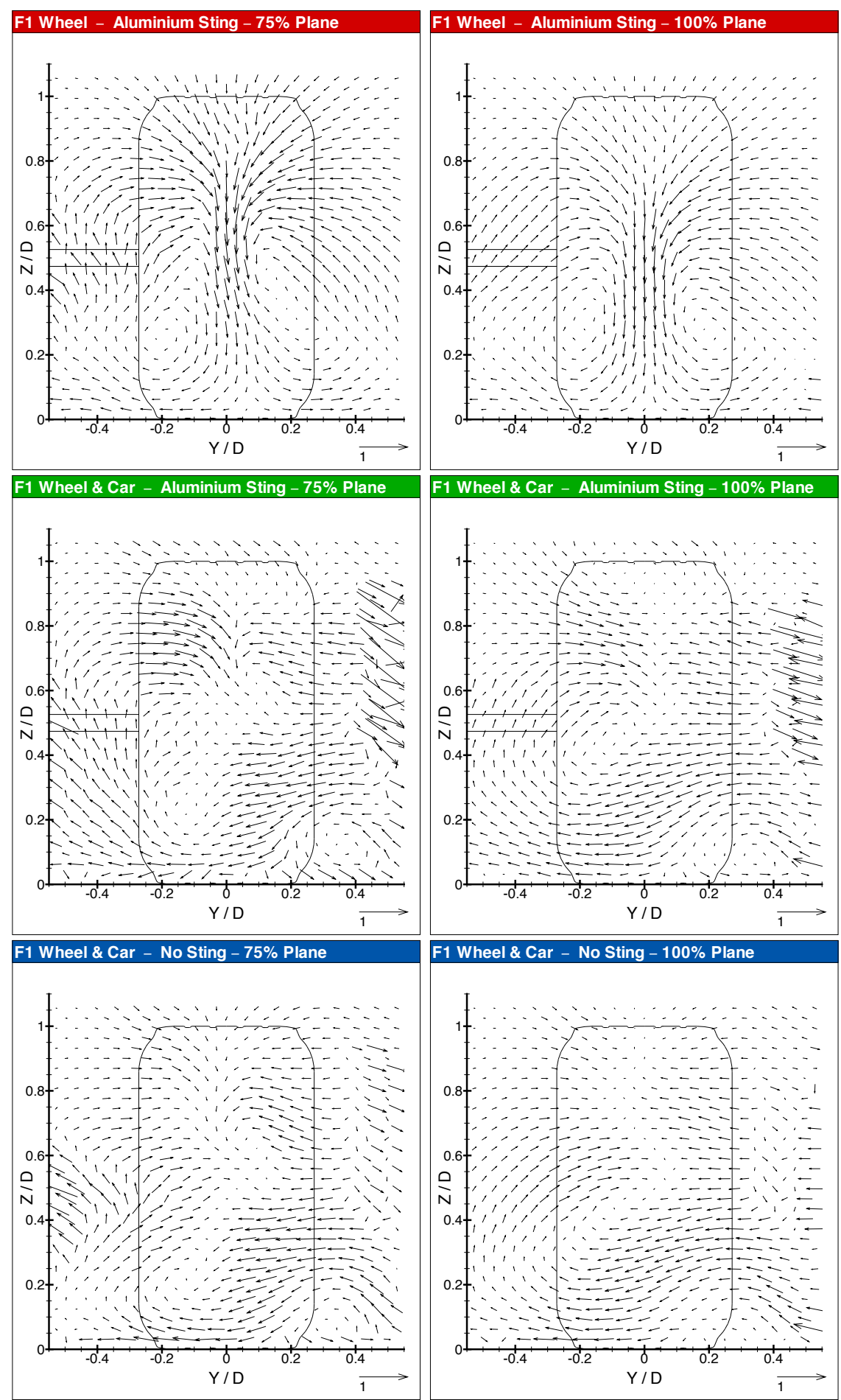

Fig. 7: In-Plane $(v-w)$ Velocity Vectors 

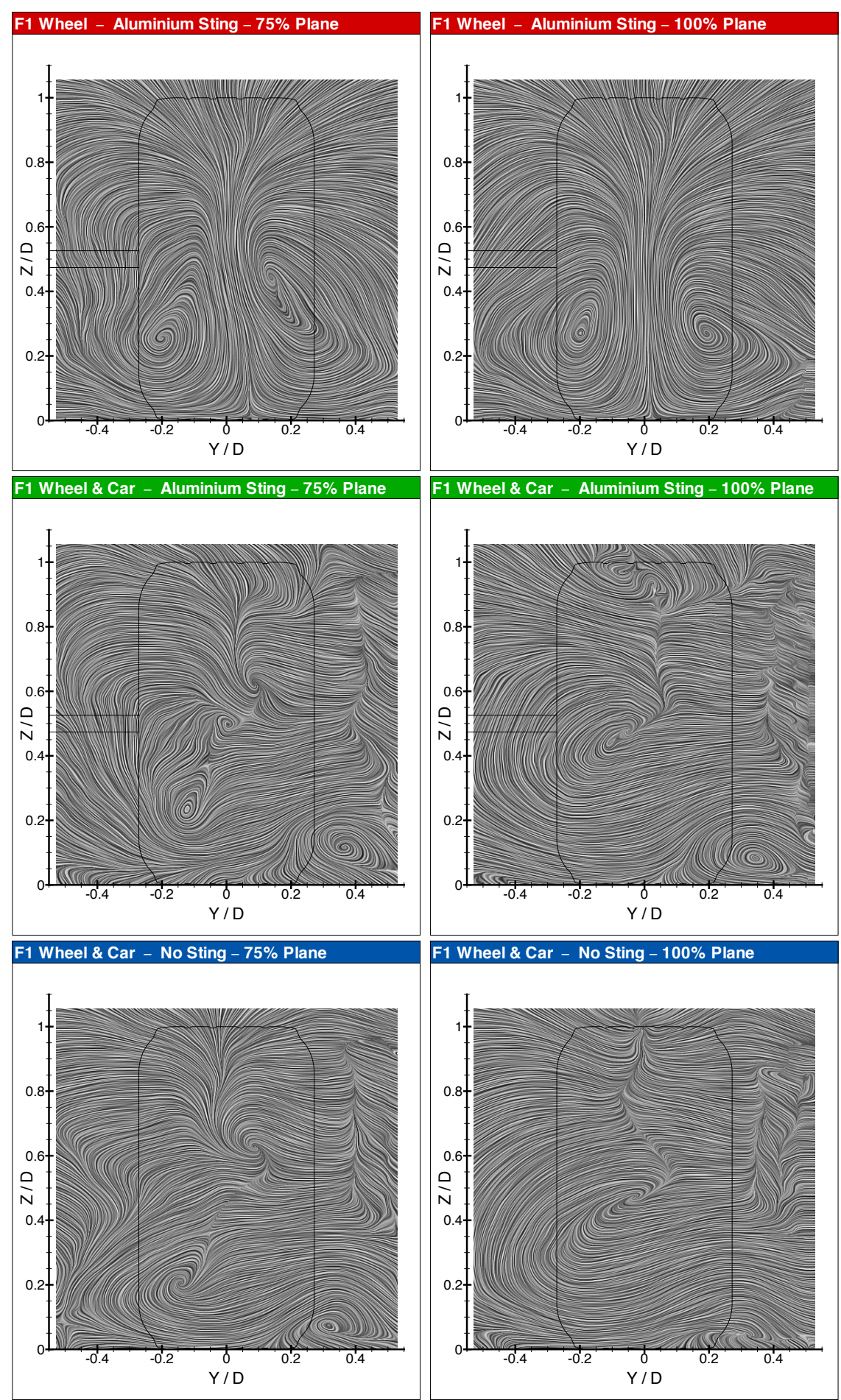

Fig. 8: In-Plane $(v-w)$ Velocity Data presented using Line Integral Convolution 
support sting on the regions of high $(>30 \%)$ turbulence intensity. Without the support sting the $0.75 \mathrm{D}$ plane has a significantly larger region of high turbulence intensity which increased diffusion and mixing such that by the next plane this configuration had much lower turbulence intensity than the others measured. Fackrell [4] observed regions of high turbulence intensity in the wake of the support strut, this is not evident from the measurements presented here.

\section{CONCLUSIONS}

Three-component velocity data were recorded in the near-wake of the front wheel of a Formula One car. Tests were carried out at 50 percent-scale, with the wheel rotating on a moving ground. All tests were conducted at a Reynolds number, based on wheel diameter, of $6.8 \times 10^{5}$. Three component configurations were investigated to determine the effect of both the car and the wheel support strut on the wheel wake.

These data were used to visualise the extent of the wake, the flow structures present and also the streamwise turbulence intensity.

The presence of the car was found to significantly alter the structure of the wheel wake when compared to an isolated wheel.

The measurements reported in this study suggest that separation from the tyre surface is much earlier in the presence of a car, leading to a taller wake. The earlier separation also reduces the entrainment effect of any upper vortices, resulting in a wider wheel wake in the upper section.

The large trailing-vortex structures previously identified for isolated wheels were not measured in the presence of the car. 

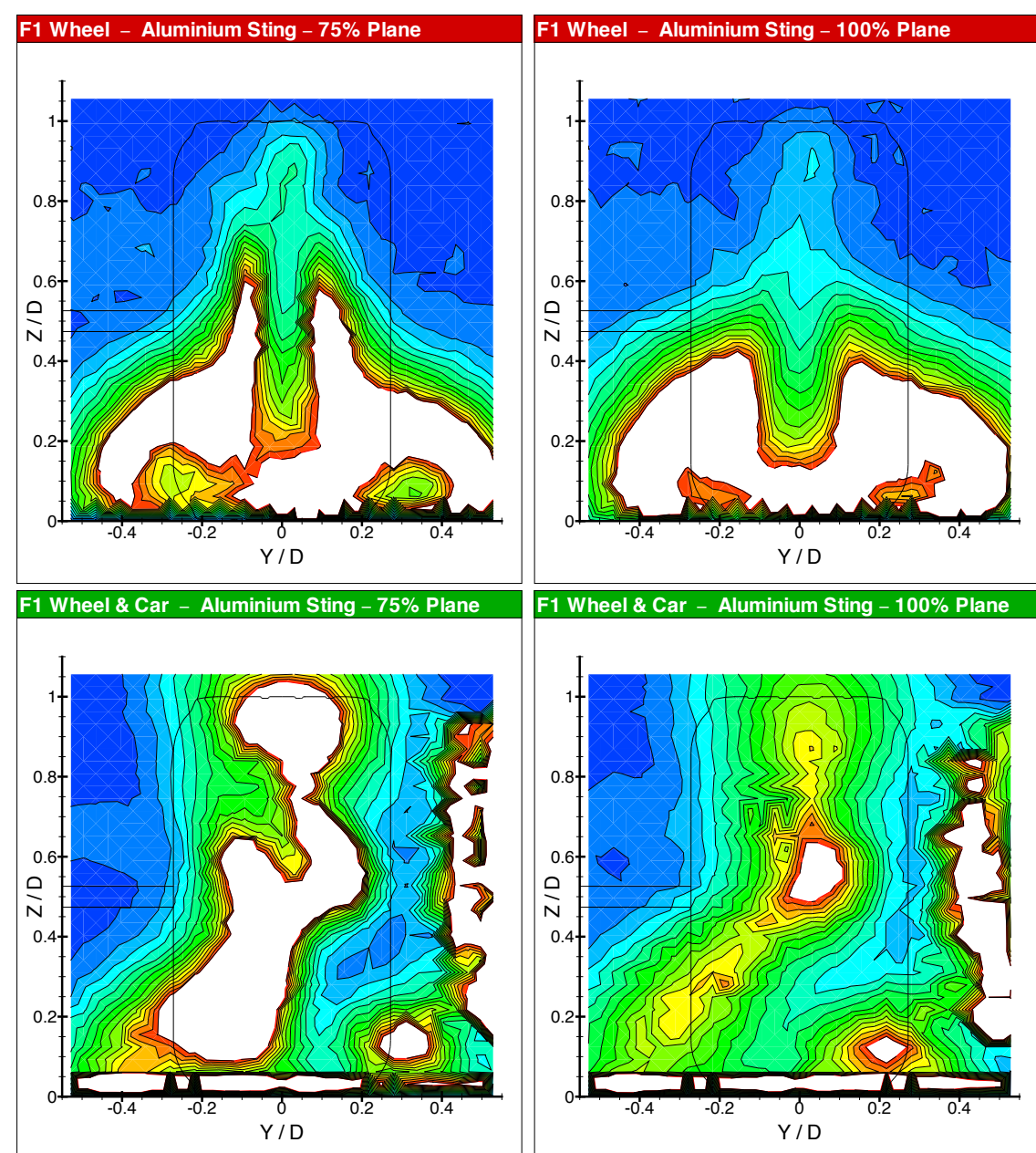

\begin{aligned} & $\mathrm{Ti}_{\mathrm{u}} \\ &$\hline 30.0 \\ & 28.0 \\ & 26.0 \\ & 24.0 \\ &-22.0 \\ &-20.0 \\ & \hline 18.0 \\ & \hline 16.0 \\ & \hline 14.0 \\ & \hline 12.0 \\ & \hline 10.0 \\ & 8.0 \\ & \hline 6.0 \\ & 4.0 \\ & 2.0 \\ & 0.0\end{aligned}
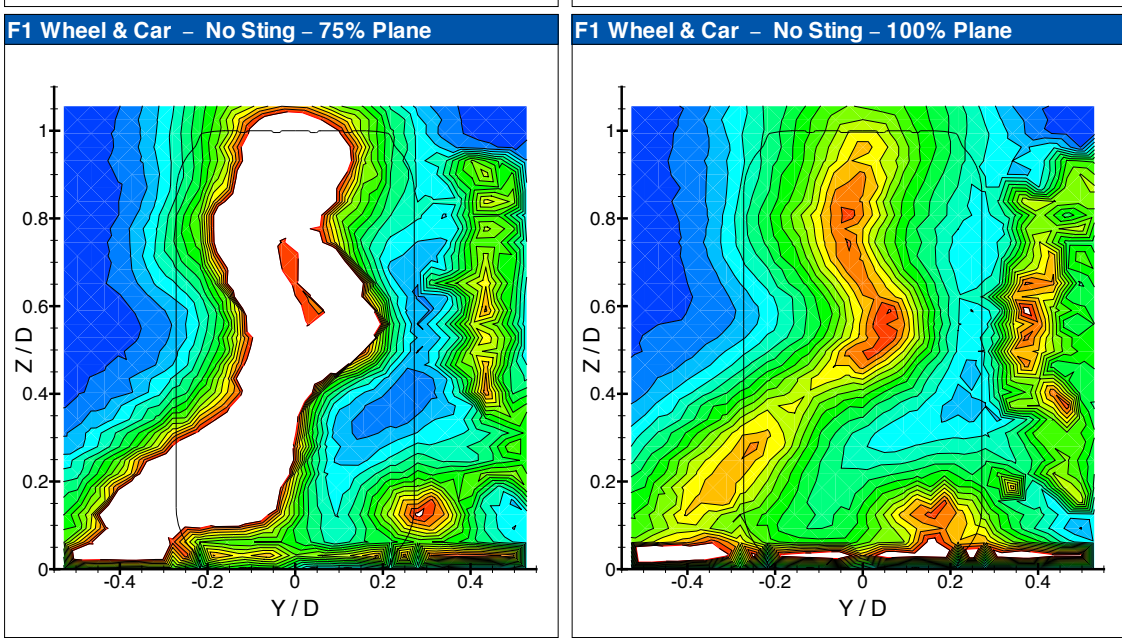

Fig. 9: Contours of Streamwise Turbulence Intensity for $x / D=0.75$ and 1.0 at three test config25 urations. 
Inspection of the in-plane and streamwise velocity data, particularly the regions of reversed flow, suggested that in the presence of the car the recirculations present may have a vertical rather than streamwise axis.

The support strut was found to have less impact in the presence of the car than previous isolated wheel measurements had suggested. However, there was a measurable effect on the turbulence intensity in the near wake. Removing the strut in the presence of the car had the effect of increasing the regions of high $(>30 \%)$ turbulence intensity at the forward measurement plane. This is likely to have increased diffusion and mixing as at one wheel diameter downstream the turbulence levels are lower than the other measured configurations.

\section{ACKNOWLEDGEMENTS}

Financial support from an Engineering and Physical Sciences Research Council CASE award and the loan of wind tunnel model components from Jaguar Racing is gratefully acknowledged.

\section{REFERENCES}

1 A. Morelli. Aerodynamics actions on an automobile wheel. In 1st Symposium on Road Vehicle Aerodynamics, Paper 5, City University, London, November 1969.

2 W. R. Stapleford and G. W. Carr. Aerodynamic characteristics of exposed rotating wheels. Report 1970/2, Motor Industry Research Association, Nuneaton, UK, 1970.

3 A. Cogotti. Aerodynamic characteristics of car wheels. Int. J. of Vehicle Design, Technological 
Advances in Vehicle Design Series, SP3, Impact of Aerodynamics on Vehicle Design, pages 173-196, 1983.

4 J. E. Fackrell. The Aerodynamics of an Isolated Wheel Rotating in Contact with the Ground. PhD thesis, University of London, UK, 1974.

5 J. E. Fackrell and J. K. Harvey. The aerodynamics of an isolated road wheel. In B. Pershing, editor, 2nd AIAA Symposium of Aerodynamics of Sports and Competition Automobiles, volume 16, pages 119-125, Los Angeles, CA, 11 May 1974. Western Periodicals Co. ISBN 0879380284.

6 J. E. Fackrell and J. K. Harvey. The flow field and pressure distribution of an isolated road wheel. In H. S. Stephens, editor, Advances in Road Vehicle Aerodynamics, pages 155-165. BHRA Fluids Engineering, Cranfield, UK, 1973.

7 J. E. Hackett, J. B. Baker, J. E. Williams, and S. B. Wallis. On the influence of ground movement and wheel rotation in tests on modern car shapes. Technical Paper 870245, Society of Automotive Engineers, Warrendale, PA, 1987.

8 P. W. Bearman, D. De Beer, E. Hamidy, and J. K. Harvey. The effect of a moving floor on wind-tunnel simulation of road vehicles. Technical Paper 880245, Society of Automotive Engineers, Warrendale, PA, 1988.

9 E. Mercker and H. Berneburg. On the simulation of road driving of a passenger car in a 
wind tunnel using a moving belt and rotating wheels. In Proceedings of 3rd International Conference on Innovation and Reliability, Florence, Italy, 8-10 April 1992.

10 E. Mercker, N. Breuer, H. Berneburg, and H. J. Emmelmann. On the aerodynamic interference due to the rotating wheels of passenger cars. Technical Paper 910311, Society of Automotive Engineers, Warrendale, PA, 1991.

$11 \mathrm{~J}$. Wildi. Wind tunnel testing of racing cars - the importance of the road simulation technique. In Royal Aeronautical Society Conference on Wind Tunnels and Wind Tunnel Testing, Loughborough, UK, September 1994.

12 R. Mueller, N. Singer, and W. Eckert. Moving belt with distributed suction in the Porsche model wind tunnel. Technical Paper 1999-01-0650, Society of Automotive Engineers, Warrendale, PA, 1999.

13 M. Hinson. Measurement of the lift produced by an isolated, rotating formula one wheel using a new pressure measurement system. MSc thesis, Cranfield University, Bedfordshire, UK, 1999.

14 A. F. Skea, P. R. Bullen, and J. Qiao. CFD simulations and experimental measurements of the flow over a rotating wheel in a wheel arch. Technical Paper 2000-01-0487, Society of Automotive Engineers, Warrendale, PA, 2000.

15 A. P. Mears, R. G. Dominy, and D. B. Sims-Williams. The flow about an isolated rotating 
wheel - Effects of yaw on lift, drag and flow structure. In Proceedings of the 4th MIRA International Vehicle Aerodynamics Conference, Warwick, UK, 16-19 October 2002.

16 A. P. Mears, R. G. Dominy, and D. B. Sims-Williams. The air flow about an exposed racing wheel. Technical Paper 2002-01-3290, Society of Automotive Engineers, Warrendale, PA, 2002.

17 A. P. Mears, S. C. Crossland, and R. G. Dominy. An investigation into the flow-field about an exposed racing wheel. Technical Paper 2004-01-0446, Society of Automotive Engineers, Warrendale, PA, 2004.

18 A. P. Mears and R. G. Dominy. Racing car wheel aerodynamics - Comparisons between experimental and CFD derived flow-field data. Technical Paper 2004-01-3555, Society of Automotive Engineers, Warrendale, PA, 2004.

19 A. Wäschle, S. Cyr, T. Kuthada, and J. Wiedermann. Flow around an isolated wheel - experimental and numerical comparison of two CFD codes. Technical Paper 2004-01-0445, Society of Automotive Engineers, Warrendale, PA, 2004.

20 J. E. Nigbur. Characteristics of the wake downstream of an isolated automotive wheel. MSc thesis, Cranfield University, Bedfordshire, UK, 1999.

21 R. D. Knowles, A. J. Saddington, and K. Knowles. Simulation and Experiments on an Isolated Racecar Wheel Rotating in Ground Contact. In Proceedings of the 4th MIRA International Vehicle Aerodynamics Conference, Warwick, UK, 16-19 October 2002. 
22 R. Knowles, A. Saddington, and K. Knowles. On the near wake of rotating, 40\%-scale Champ Car wheels. SAE 2002 Transactions - Journal of Passenger Cars: Mechanical Systems, 6: 2245-2253, 2002. doi: 10.4271/2002-01-3293.

23 R. D. Knowles. Monoposto Racecar Wheel Aerodynamics: Investigation of Near-Wake Structure \& Support Sting Interference. PhD thesis, Cranfield University, Shrivenham, UK, 2005.

24 A. J. Saddington, R. D. Knowles, and K. Knowles. Laser Doppler anemometry measurements in the near-wake of an isolated Formula One wheel. Experiments in Fluids, 42(5):671-681, March 2007. ISSN 0723-4864. doi: 10.1007/s00348-007-0273-7.

25 Emin Issakhanian, Chris J. Elkins, Kin Pong Lo, and John K. Eaton. An Experimental Study of the Flow Around a Formula One Racing Car Tire. Journal of Fluids Engineering, 132(7): 071103, 2010. doi: 10.1115/1.4001880.

26 A. F. Skea, P. R. Bullen, and J. Qiao. The use of CFD to predict the air flow around a rotating wheel. In 2nd MIRA International Conference on Vehicle Aerodynamics, Birmingham, UK, October 1998.

27 L. Axon. The Aerodynamic Characteristics of Automobile Wheels - CFD Prediction and Wind Tunnel Experiment. PhD thesis, Cranfield University, Bedfordshire, UK, September 1999.

28 B. Basara, D. Beader, and V. P. Przulj. Numerical simulation of the air flow around a rotating wheel. In 3rd MIRA International Conference on Vehicle Aerodynamics, Rugby, UK, 18-19 October 2000. 
29 James McManus and Xin Zhang. A Computational Study of the Flow Around an Isolated Wheel in Contact With the Ground. Journal of Fluids Engineering, 128(3):520, 2006. ISSN 00982202. doi: $10.1115 / 1.2175158$.

30 John Axerio, Gianluca Iaccarino, and Emin Issakhanian. Computational and Experimental Investigation of the Flow Structure and Vortex Dynamics in the Wake of a Formula 1 Tire. SAE Technical Paper 2009-01-0775, Society of Automotive Engineers, 2009.

31 John Axerio-Cilies, Emin Issakhanian, Juan Jimenez, and Gianluca Iaccarino. An Aerodynamic Investigation of an Isolated Stationary Formula 1 Wheel Assembly. Journal of Fluids Engineering, 134(2):021101, 2012. ISSN 00982202. doi: 10.1115/1.4005768.

32 M. V. Finnis, K. Knowles, R. Lewis, D. Pitchforth, and A. J. Reynard. A New 3/4 Open-Jet Wind Tunnel For Racing Car Aerodynamic Testing. In 3rd MIRA International Conference on Vehicle Aerodynamics, Rugby, UK, 18-19 October 2000.

33 R. P. Dring. Sizing criteria for laser anemometry particles. ASME Journal of Fluids Engineering, 104:15-17, March 1982.

34 P. Buchave, W. K. George Jr, and J. L. Lumley. The measurement of turbulence with the laser-doppler anemometer. Annual Review of Fluid Mechanics, 11:443-503, 1979.

35 L. H. Benedict and R. D. Gould. Towards better uncertainty estimates for turbulence statistics. Experiments in Fluids, 22(2):129-136, December 1996.

$36 \mathrm{H}$. Nobach. A global concept of autocorrelation and power spectral density estimation from 
LDA data sets. In 10th International Symposium on Applications of Laser Techniques to Fluid Mechanics, Instituto Superior Técnico, Lisbon, Portugal, 10-13 July 2000.

37 R. D. Knowles, M. V. Finnis, A. J. Saddington, and K. Knowles. Planar visualization of vortical flows. IMechE Part G: Journal of Aerospace Engineering, 220(6), 2006. doi: 10.1243/09544100JAERO75.

38 M. A. van den Berg. Aerodynamic Interaction of an Inverted Wing with a Rotating Wheel. PhD thesis, University of Southampton, March 2007. 